Supporting Information

\title{
Low-Temperature Vibrational Energy Transport via PEG Chains
}

Robert T. Mackin, $\stackrel{+}{+}$ Tammy X. Leong, $+\underset{+}{+}$ Natalia I. Rubtsova, Alexander L. Burin, Igor V. Rubtsov*

Department of Chemistry, Tulane University, New Orleans, Louisiana 70118, United States *corresponding author: irubtsov@tulane.edu

† These authors contributed equally.

\section{Table of Contents}

1. FTIR spectra of azPEG2 at various temperatures

2. NN/CO cross-peak amplitude as a function of temperature

3. Experimental methods

4. Modeling

5. References

\section{FTIR Spectra at Various Temperatures}
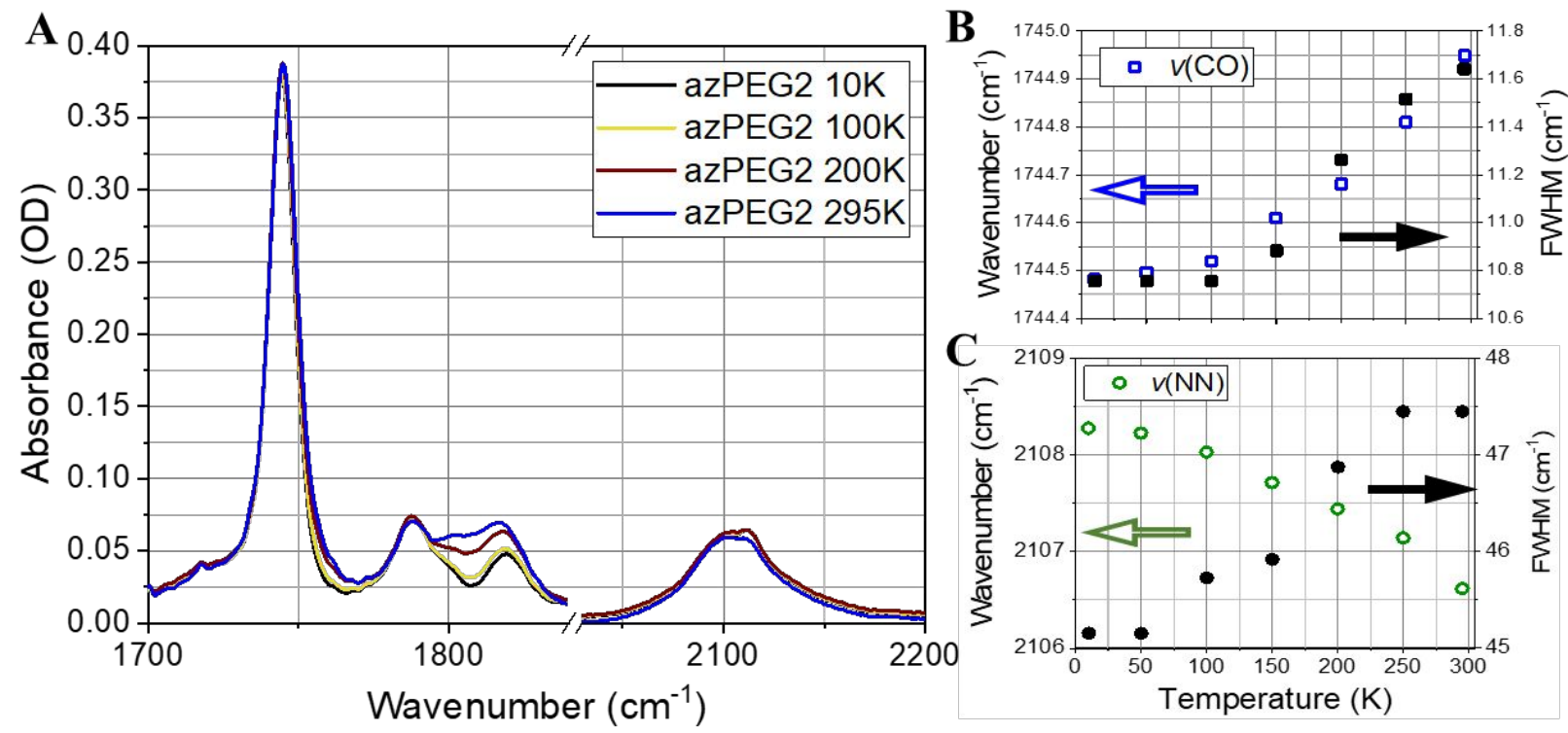

Figure S1. Temperature-dependent FTIR spectra (A) of azPEG2-NHS/PS film after PS subtraction. $(\mathrm{B}, \mathrm{C})$ The changes in the central frequency (left axis) and the full-width half max (FWHM, right axis) for the $\mathrm{CO}$ and $\mathrm{NN}$ modes as a function of temperature are provided in (B) and $(\mathrm{C})$, respectively. The open and filled arrows correspond to the open and filled data point and refer to the axis for each data set. 


\section{NN/CO Cross Peak Amplitude as a Function of Temperature}

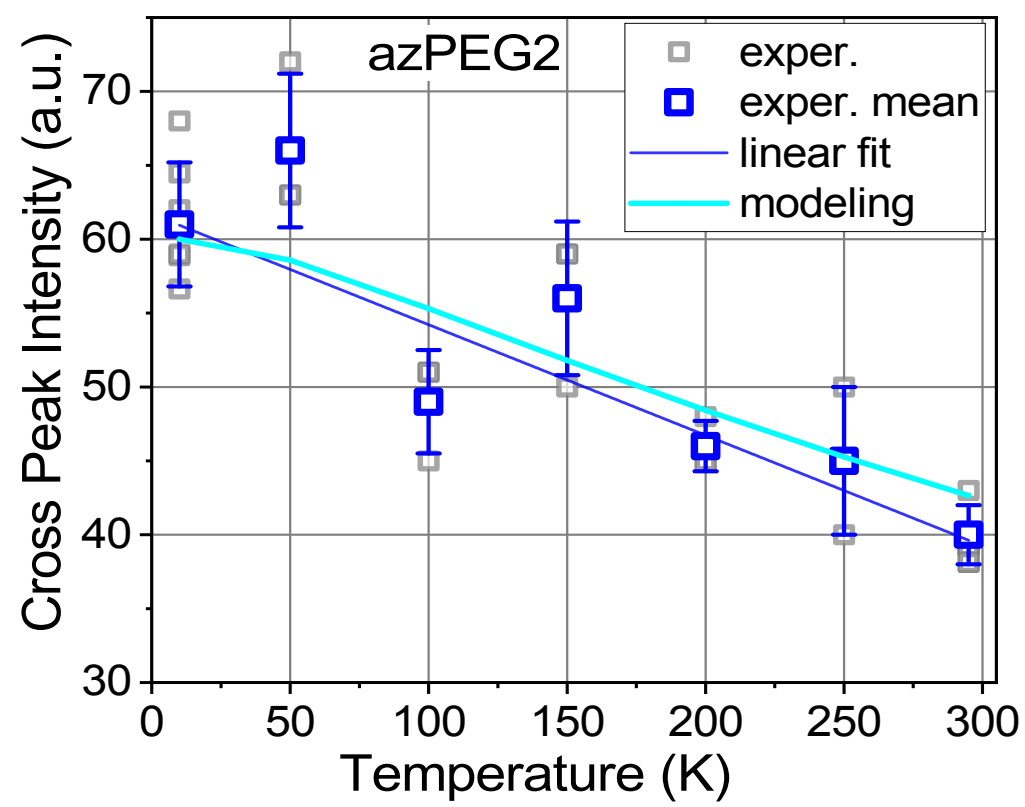

Figure S2. Cross peak intensity as a function of temperature. The ca. $50 \%$ increase in intensity as temperature is decreased from 295 to $10 \mathrm{~K}$ suggests an increase in vibrational energy transport efficiency at lower temperatures. The modeling is shown with cyan (see also Fig. S7).

\section{Experimental Methods}

Sample Preparation. The azPEG $n$ compounds, $n=0,2,4,8$ were purchased from Synthonix, and the polystyrene (PS, average molecular weight of $\mathrm{M}_{\mathrm{w}}=35000 \mathrm{~g} / \mathrm{mol}$ ) was purchased from Sigma-Aldrich (331651). Note that the compounds with $n=2,4,8$ have two bridging carbon atoms on the chain outside of the PEG units, the azPEG0 compound contains just three methylene groups in its chain. Thin films for each of the azPEGn/PS samples were produced through an extensive process. First, the azPEG $n$ and PS were co-dissolved in $\mathrm{CHCl}_{3}$ targeting the azPEG $n$ concentration in dry PS of $16.6 \mathrm{mM}$. This solution was then poured into an aluminum tray and placed in an oven to remove the solvent, resulting in a homogeneous mixture of azPEGn/PS. A portion of the mixture was placed in a $13 \mathrm{~mm}$ die/anvil set (International Crystal Laboratories), which was heated to $100^{\circ} \mathrm{C}$ over an hour, and then pressed in a hydraulic press for a minute to a pressure of $87 \mathrm{MPa}(12,730 \mathrm{psi})$. After pressing the film, it cooled to room temperature before removing from the anvils. Films of about $200 \mu \mathrm{m}$ thickness with an optical density of ca. 0.7 at $v_{\mathrm{CO}}\left(1740 \mathrm{~cm}^{-1}\right)$ were made for each azPEGn sample. A high optical quality of the films was achieved (Figure S3).

FTIR Measurements. Linear FTIR measurements were performed in transmission mode with a Vertex 80 spectrometer (Bruker). When examining the temperature dependence, a series of measurements were performed. First, the background was recorded at each temperature. These 
measurements were followed by a pure PS film spectrum, also measured at each point in the temperature range. Finally, the azPEGn/PS film sample was measured at each temperature. The pure PS film spectrum was subtracted from the azPEGn/PS linear spectrum resulting in the pure $\mathrm{azPEG} n$ spectrum at each temperature.

2DIR Measurements. A fully-automated dual-frequency three-pulse 2DIR spectrophotometer with heterodyned detection is described in detail elsewhere. ${ }^{1-2}$ Briefly, the spectrometer is powered by a Ti:sapphire laser (Libra, Coherent) which produces $1.5 \mathrm{~W}$ power at $1 \mathrm{kHz}$ repetition rate and $80 \mathrm{fs}$ pulse duration. Mid-IR pulses of ca. $150 \mathrm{fs}$ duration are generated with a dual optical parametric amplifier and difference frequency generation units (Palitra-Duo, Quantronix). Waiting time signals up to $60 \mathrm{ps}$ are collected in the time domain and Fourier transformed to produce the 2D spectrum. One dimensional waiting-time kinetics plots are generated for each diagonal and cross peak by integrating the 2D spectrum in a region around the peaks. At least three waiting-time measurements were recorded for each length of azPEG $n$ at each temperature.

Cryostat Measurements. The low-temperature measurements were performed using a closedloop helium cryostat (Advanced Research Systems, Inc.) capable of reaching temperatures of $<10 \mathrm{~K}$. Dual temperature sensors of the instrument guarantee limited temperature fluctuations $(<0.3 \mathrm{~K})$ for the duration of the measurements. The cryostat was integrated into the sample cell position for both the FTIR and 2DIR measurements.

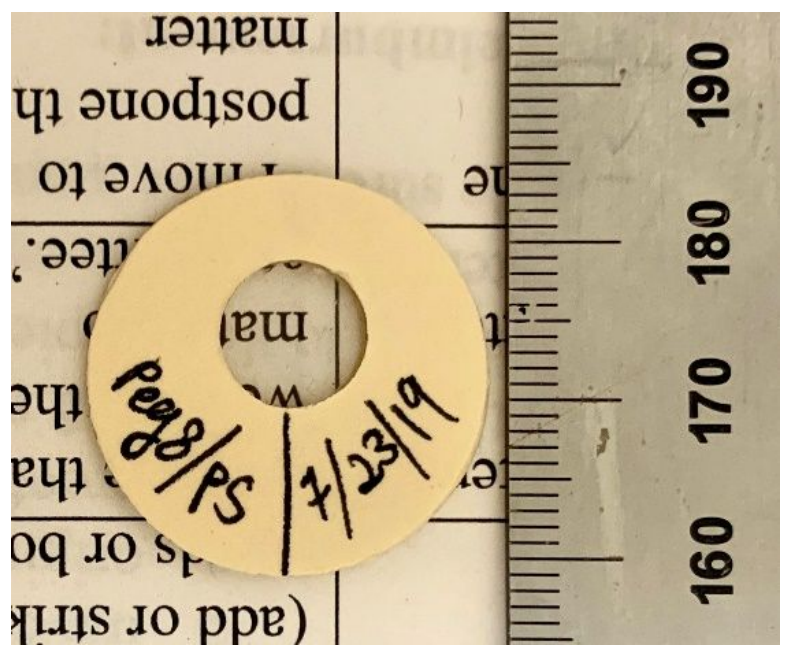

Figure S3. High optical quality azPEG8/PS film photo. The film is held in place between two pieces of cardstock. The optical quality is such that the letters behind it are not distorted. 


\section{Modeling}

\section{A. Formalism}

The density matrix formalism ${ }^{3}$ is used to describe the transport from the tag to the reporter site through $N$ chain sites (Figure S4). The linear transport time dependence on the distance suggests ballistic nature of the transport; we assume that one specific transport band is involved, formed by $N$ vibrational states within the chain. Since the number of excitations per molecule does not exceed unity, we used $N+2$ basic states, which included tag, reporter and $N$ chain sites. Consequently, the density matrix has $(N+2)^{2}$ elements. We assume that coherence takes place only within the chain while the coupling of chain to tag and reporter sites are entirely incoherent, so that the density matrix $\hat{\rho}$ is reduced to two diagonal elements $\rho_{00}$ and $\rho_{N+1, N+1}$ for the tag and reporter sites, respectively, and $N^{2}$ matrix with $\rho_{a b}$ elements for chain sites $a, b=1,2$, ... N. The

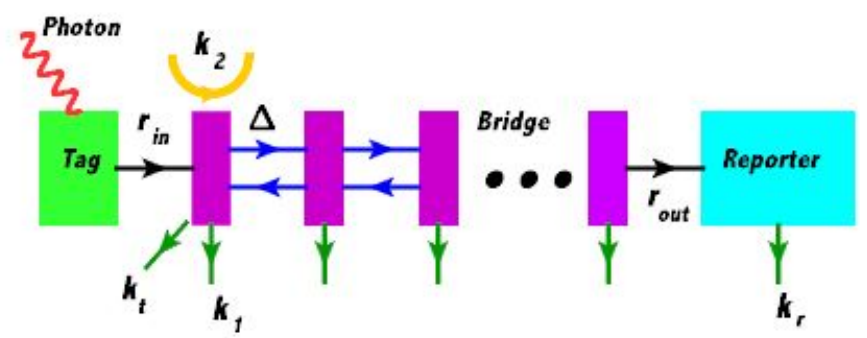

Figure S4. Schematic description of energy transport through in azPEGn compounds.

equations for the density matrix evolution within the chain $(1<a, b<N)$ can be written similarly to the Bloch equations ${ }^{3-4}$ with relaxation and decoherence rates added:

$$
\frac{d \rho_{a b}}{d t}=i \Delta\left(\rho_{a+1, b}+\rho_{a-1, b}-\rho_{a, b+1}-\rho_{a, b-1}\right)-\left(k_{1}+k_{2}\left(1-\delta_{a b}\right)\right) \rho_{a b}
$$

Here $\delta_{a b}$ is the Kronecker symbol ( $\delta_{a b}=1$ for $a=b$ and zero otherwise), we assumed that there is no population of the excited state of the chain band at the equilibrium. The relaxation $\left(k_{1}\right)$ is considered as fully irreversible assuming that after the band state decay most of the energy is dissipated either to the surroundings or to delocalized low energy modes, which cannot significantly influence the reporter site.

The equations for off-diagonal matrix elements with $a$ or $b$ at the edge ( 1 or $N$ ) can be written similarly to Eq. (S1) omitting the non-existing 0 and $N+1$ site indices. There is no connection of these off-diagonal elements to the tag or reporter sites because we assumed the lack of coherence between them and chain sites. The equations for diagonal elements in the first and $N^{\text {th }}$ sites should include connections to the tag and reporter in the form

$$
\frac{d \rho_{11}}{d t}=i \Delta\left(\rho_{2,1}-\rho_{1,2}\right)-\left(k_{1}+k_{2}\right) \rho_{11}+r_{i n} \rho_{00}-k_{1 t} \rho_{11}
$$




$$
\frac{d \rho_{N N}}{d t}=i \Delta\left(\rho_{N-1, N}-\rho_{N, N-1}\right)-\left(k_{1}+k_{2}+r_{o u t}\right) \rho_{N N},
$$

where $r_{\text {in }}$ is the transition rate from the tag site to the adjacent chain site and rout is the transition rate from the chain to the reporter site. We also include the decay channel $\left(k_{1 t}\right)$ for the matrix element $\rho_{11}$ from the first chain site towards the tag site. Finally, the equations for the tag and reporter populations should be added as following

$$
\begin{aligned}
& \frac{d \rho_{00}}{d t}=-r_{\text {tag }} \rho_{00}, \\
& \frac{d \rho_{N+1, N+1}}{d t}=r_{\text {out }} \rho_{N N}-k_{r} \rho_{N+1, N+1} .
\end{aligned}
$$

The rate $r_{\text {tag }}$ is the decay rate of the tag state. It can contain several channels including the formation of the wavepacket in the chain $r_{\text {in }}$. Consequently, one would expect $r_{\text {in }} \leq r_{\text {tag }}$. The rate $k_{r}$ accounts for the irreversible decay of the excited reporter state.

The initial condition suggests the excitation of the tag at time $t=0$, meaning that $\rho_{00}(0)=$ 1 , while all other elements of the density matrix are zeros at zero time. The cross peak is determined by the time dependent population of the reporter site $P_{N+1, N+1}(t)$. We define the transport time, $t_{\max }$, as the time when this population approaches its maximum. The first site of the chain, $a=1$, is excited by energy transfer from the tag with the rate $r_{\text {in }}$. The energy is transferred between the chain sites to the final site, $k=N$, and to the reporter site with the rate $r_{2}$, where it relaxes with the rate $r_{3}$ due to dissipation to the PS matrix (Figure S4). The transitions between the tag and alkyl chain and the alkyl chain and reporter are considered as incoherent and irreversible. The energy transport through the molecule is determined by the time evolution of the density matrix. At zero time $(t=0)$, all elements of the density matrix $\rho_{a b}(a, b=1,2, \ldots N)$ equals zero. Further evolution of density matrix can be described by the equation

$$
\begin{aligned}
& \frac{d \rho_{a b}}{d t}=-\left(\frac{1-\delta_{a b}}{T_{2}}-\frac{1}{T_{1}}\right) \rho_{a b}+i\left(1-\delta_{a, n}\right) \Delta \rho_{a+1, b}+i\left(1-\delta_{a, 1}\right) \Delta \rho_{a-1, b}- \\
& -i\left(1-\delta_{b, n}\right) \Delta \rho_{a, b+1}-i\left(1-\delta_{b, 1}\right) \Delta \rho_{a, b-1}+\delta_{a b} \delta_{a 1} r_{1} e^{-r_{1} t}
\end{aligned}
$$

The cross-peak intensity is determined by the probability of the reporter occupation $P_{r}(t)$ that obeys the equation

$$
\frac{d P_{r}}{d t}=r_{2} \rho_{n n}-r_{3} P_{r}
$$

The time $t_{\max }$ where the probability of the reporter occupation reaches maximum was compared to the experimentally observed transport time, while the occupation probability of the 
reporter at the maximum $P_{r}\left(t_{\max }\right)$ determines the associated cross-peak intensity that was also compared to the experiment.

\section{B. Evaluation of parameters and their temperature dependences}

The problem contains many unknown parameters including the chain coupling strength $\Delta$, relaxation and decoherence rates $k_{1}$ and $k_{2}$, tag-associated decay and initiation rates $r_{\text {tag }}$ and $r_{\text {in }}$ and reporter associated transition and decay rates $k_{\text {out }}$ and $k_{r}$. All these rates can show some temperature dependence; most probably the rates should increase with increasing temperature. Based on the experimental data and expectations, we choose an expected behavior for each of the parameters and run Monte-Carlo optimization to fit the experimental data (see next section). We begin our consideration with the chain associated parameters $\Delta, k_{1}$ and $k_{2}$. Note that the modeling was performed for the three compounds containing PEG units $(n=2,4,8)$ to eliminate differences in the energy entry into and exit from the chain possible for azPEG0.

It is natural to expect the bandwidth, $\Delta$, to be approximately temperature independent since it is determined by harmonic interaction while anharmonic corrections to the force constants are weak at any temperature under consideration. ${ }^{5}$ The relaxation takes place with the assistance of the PS matrix that consumes the excess of energy due to the continuity of its spectrum. This process takes place even at zero temperature. Yet the rate of this process can depend on the temperature. For instance, if it includes the emission of the vibrational quantum with frequency $\omega_{\mathrm{s}}$ to the PS matrix continuum then the process rate acquires the temperature dependence in the form

$$
k_{1}=k_{10} /\left(1-e^{-\frac{\hbar \omega_{s}}{k_{\mathrm{B}} T}}\right)
$$

Typical energy, $\hbar \omega_{s}$, is determined by a phonon bandwidth of the PS matrix that is comparable or larger than the thermal energies $k_{\mathrm{B}} T$ under consideration. Therefore, all relaxation rates are not expected to undergo major changes within the range of experimental temperatures.

According to the experiment, the dependence of the cross-peak intensity on the chain length is rather weak (Fig. S2). The cross-peak intensity was previously found to decay nearly exponentially with the decay length of ca. $16 \AA .{ }^{6}$ For the sake of simplicity, we set $k_{1}$ to zero, which significantly narrows down the choice of other parameters. With this specific choice we can obtain a good fit for the experimental data as described below.

The decoherence rate $k_{2}$ is expected to be strongly temperature dependent. This is because the decoherence is associated with the energy fluctuations disappearing at zero temperature. The decoherence can be associated with anharmonic interactions of vibrational band modes responsible for the energy transport with the low frequency thermal phonons belonging to either PS matrix or the molecule itself. The coupling of molecular vibrations to the matrix is weaker than to those of the molecule because of the non-covalent coupling between them. Also the decoherence rate due to $3 \mathrm{D}$ phonons is expected to be very weak due to their small density of states at least at low temperatures. ${ }^{7-9}$ The situation is different for one- 
dimensional acoustic vibrations of the molecule itself. Following the consideration of Ref. 8 one can see that no decoherence can be expected for the optical phonon band due to their third order anharmonic interaction with longitudinal acoustic modes due to the system translational invariance. The fourth order interaction with them results in the $T^{3}$ dependence of the decoherence rate on temperature. However, one should also consider the interaction with one dimensional transverse phonons. The associated decoherence rate can be obtained extending the calculations of Ref. 8 to that specific case assuming the transverse vibrational energy density for low frequency, long wavelength phonons to be proportional to $\left(\partial^{2} u_{\mathrm{tr}} \partial x^{2}\right)^{2}$ and interaction of optical phonon with them to $\partial^{2} u_{\mathrm{tr}} \partial x_{r}{ }_{r}$ (see e. g. Ref. 11) where $\mathrm{u}_{\mathrm{tr}}$ stands for transverse displacement of atoms and $x$ represents the coordinate along the polymer chain. These calculations result in the $k_{2}(T) \sim T^{2}$ dependence, which originates from larger expected decoherence rates for transverse phonons at small temperatures compared to that for longitudinal phonons. Therefore, we used this functional form to fit the experimental data.

\section{Tag associated rates}

Here we discuss the rates associated with the tag decay $\left(r_{\text {tag }}\right)$, wavepacket initiation $\left(r_{\text {in }}\right)$ and the decay rate $r_{t}$ of the chain site excitation towards the tag. The tag decay rate has been measured for three temperatures of 10, 150 and 290K (see Fig. 4). We were not able to fit these data with Eq. (6), therefore we used the composed expression, $r_{\mathrm{tag}}=r_{1}+r_{2}\left(1-e^{T_{0} / T}\right)$ with $r_{1}$ $=0.5 \mathrm{ps}^{-1}, r_{2}=0.11 \mathrm{ps}^{-1}$ and $T_{0}=153 \mathrm{~K}$. This equation has been used to extrapolate the experimental observations to other temperatures where cross peak has not been measured. To separate the rate for the wavepacket formation $r_{\text {in }}$ from other decay processes we assumed that it is defined by the temperature independent part $r_{1}$ of the overall tag decay rate. This assumption is made to attain the maximum increase in the chain excitation yield with decreasing the temperature. Only after making this assumption with an additional assumption for temperature dependent reporter associated rates (see next section), we were able to model the $50 \%$ raise of the cross-peak intensity with decreasing the temperature for azPEG2, (see Fig. S2). We set the decays rate $r_{t}$ to be constant determined by the Monte Carlo simulations.

\section{Reporter associated rates}

Considering the transition rates from chain to reporter $k_{\text {out }}$ and reporter decay rate $k_{r}$ we assumed that the first is temperature independent, while the second rate shows the dependence given by Eq. (S6). Under this assumption the temperature decrease leads to a longer cross-peak times at low temperature because of its slower decay $\left(k_{\mathrm{r}}\right)$ that is consistent with the temperature dependence of cross-peak times. Yet, the cross-peak maximum intensity will be larger at lower temperatures in agreement with the experimental observations.

\section{E. Monte-Carlo analysis of optimum fit parameters}


The remaining problem is to define the six optimum fitting parameters including vibrational coupling $\Delta$, relaxation and decoherence rates within the chain $\left(k_{t}\right.$ and $\left.k_{20}\right)$, the rate of the energy transport between the chain and reporter $r_{\text {out }}$ and reporter relaxation rate pre-factor $k_{r} 0$ and the temperature dependence parameter $T_{0}$ for $r_{\text {tag. }}$.

Applying the Monte-Carlo search algorithm described in Ref. ${ }^{10}$ minimizing the root mean squared difference between all experimental and theoretical cross peak data we obtained the following parameters: $\Delta=1.03 \mathrm{ps}^{-1}, k_{t}=1.76 \mathrm{ps}^{-1}, k_{20}=0.27 \mathrm{ps}^{-1}, r_{\text {out }}=1.98 \mathrm{ps}^{-1}, k_{r 0}=0.038$ $\mathrm{ps}^{-1}$ and $T_{0}=727 \mathrm{~K}$. The estimate for the coupling parameter $\Delta=1.03 \mathrm{ps}^{-1}$ is consistent with the observed velocity of the energy transport of ca. $7 \AA / \mathrm{ps}$. Indeed, according to the analytical solution obtained earlier ${ }^{6}$ the ballistic energy transport velocity can be estimated as the maximum velocity $V_{\max }=2 \Delta a=9 \AA / \mathrm{ps}$, where $a=4.36 \AA$ is a PEG chain period. The actual observed velocity is between the maximum one and the center of mass velocity ${ }^{4}$ given by $2 v_{\mathrm{m}} / \pi=5.7 \AA / \mathrm{ps}$ (Fig. 3C). The origin of the reduction of transport velocity with increasing the temperature is discussed in the next section.

The rate estimates are all consistent with typical transport times of a few picoseconds, except for the very slow decay rate of reporter $k_{\mathrm{r}}$ that probably needs a strong participation of the PS matrix and large decays of chain states at edges (around $2 \mathrm{ps}^{-1}$ ) that can be an artifact of our oversimplified model where we set $k_{1}=0$. The estimate for the temperature $T_{0}$ in the expression for $r_{\text {tag }}$ suggests that the reporter vibrational energy can be consumed by some collective optical modes of the PS matrix.

Figure 3A shows a comparison of the theory predictions with the experimental data for cross peak maximum times. The agreement between experiment and theory is within the experimental error. The cross-peak amplitude temperature dependence is shown in Figure S5. Both the reduction of the cross-peak amplitude with increasing the temperature by the factor of 1.5 for azPEG2 and the amplitude dependence on the molecular length at room temperature is consistent with the experiment.

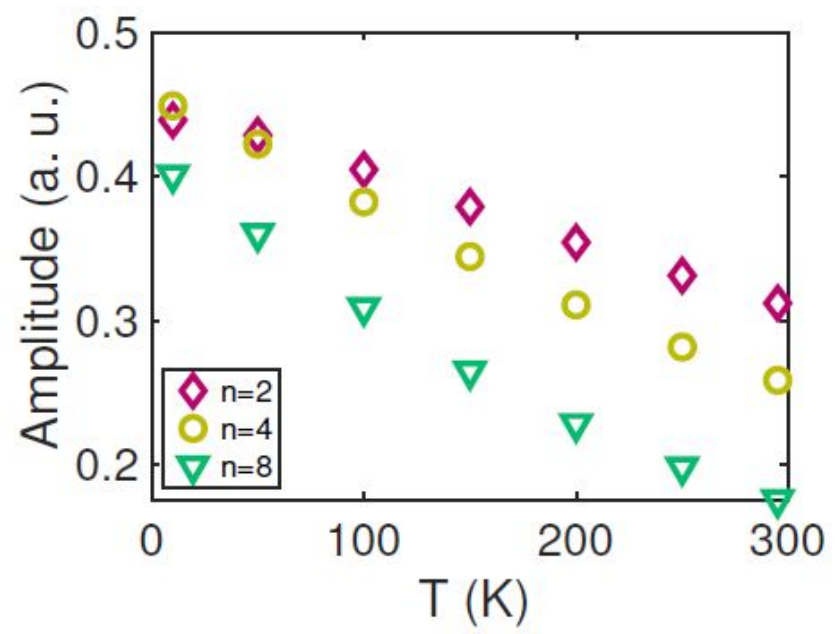

Figure S5. Monte-Carlo modeling of the temperature dependence for the cross-peak amplitudes for azPEG2, azPEG4, and azPEG8. 


\section{F. The origin of the velocity temperature dependence}

For the chosen set of parameters one can expect, based of Ref. 4, that at room temperature $(T=295 \mathrm{~K})$ for the transport along an infinite chain the ballistic transport regime takes place at distances less than $l=V / k_{2}=28 \AA$ from the starting point, while at longer distances diffusive transport is expected. The crossover distance $l$ at room temperature is comparable to the PEG6 chain length. Therefore, at high temperatures in azPEG8, one can expect that the transport is given by the complicated combination of ballistic and diffusive transport mechanisms. The diffusive transport slows down with the temperature increase because the diffusion coefficient is inversely proportional to the decoherence rate, ${ }^{3}$ which in turn is increased at higher temperatures resulting in slower transport.

In the ballistic regime the decoherence can lead to an opposite effect, associated with the shift of $t_{\mathrm{m}}$ in response to a change in the decoherence and/or relaxation at the reporter site. One can qualitatively explain this effect by expanding the waiting time dependence of the cross-peak amplitude near its maximum as $I(t)=\exp \left(-A\left(t-t_{0}\right)^{2}-k_{2} t\right)$ (see Ref. 4), where the time $t=t_{0}$ describes the maximum in the absence of decoherence. The position of the maximum, $t_{\mathrm{m}}$, can be found by setting the time derivative of $I(t)$ to zero, yielding $t_{m}=t_{0}-k_{2} /(2 A)$. At low temperatures the decoherence times, $1 / k_{2}$, increases, which affecting $t_{\mathrm{m}}$ and the apparent transport velocity. This effect results in a small reduction of the transport speed determined via modeling for the 10 $\mathrm{K}$ temperature, compared to that at $50 \mathrm{~K}$ (Fig. 3C). More detailed experiments in the low frequency region $(<100 \mathrm{~K})$ are needed to gain more insight on this contribution. At higher temperature the diffusion contribution becomes significant which is probably the origin of the observed increase of the energy transport velocity with the temperature for $T>100 \mathrm{~K}$.

\section{References}

(1) Leger, J. D., et al. Fully Automated Dual-Frequency Three-Pulse-Echo 2dir Spectrometer Accessing Spectral Range from 800 to 4000 Wavenumbers. Rev. Sci. Instrum. 2014, 85, 083109.

(2) Nyby, C. M.; Leger, J. D.; Tang, J.; Varner, C.; Kireev, V. V.; Rubtsov, I. V. Mid-Ir Beam Direction Stabilization Scheme for Vibrational Spectroscopy, Including Dual-Frequency 2dir. Opt. Express 2014, 22, 6801-9.

(3) Kurnosov, A. A.; Rubtsov, I. V.; Burin, A. L. Fast Transport and Relaxation of Vibrational Energy in Polymer Chains. J. Chem. Phys. 2015, 142, 011101/1-011101/4.

(4) Rubtsova, N. I.; Kurnosov, A. A.; Burin, A. L.; Rubtsov, I. V. Temperature Dependence of the Ballistic Energy Transport in Perfluoroalkanes. J. Phys. Chem. B 2014, 118, 8381-8387.

(5) Barone, V. Anharmonic Vibrational Properties by a Fully Automated Second-Order Perturbative Approach. J. Chem. Phys. 2005, 122, 14108.

(6) Yue, Y., et al. Band-Selective Ballistic Energy Transport in Alkane Oligomers: Toward Controlling the Transport Speed. J. Phys. Chem. B 2015, 119, 6448-56.

(7) Kagan, Y. Quantum Diffusion in Solids. J. Low Temp. Phys. 1992, 87, 525-569.

(8) Kagan, Y.; Maksimov, L. A. Effect of Interparticle Interaction on Localization in a Nonideal Crystal with a Narrow Band. J. Exper. Theor. Phys. 1985, 88, 992-1000.

(9) Yu, K.; P.N.V. JETP Letters 1986, 43. 
(10) Burin, A. L.; Leveritt, J. M. I. I. I.; Ruyters, G.; Schoetz, C.; Bazrafshan, M.; Fassl, P.; von Schickfus, M.; Fleischmann, A.; Enss, C. Low-Temperature Dipolar Echoes in Amorphous Dielectrics: Significance of Relaxation and Decoherence Free Two-Level Systems. EPL 2013, 104, 57006.

(11) Landau, L.D.; Pitaevskii, L. P.; Kosevich, A. M.; Lifshitz, E.M., Theory of Elasticity: Volume 7 (Theoretical Physics) 3rd Edition, Elsevier, 1986. 